\title{
Integration of Primary Health Care \& Wellness Services Into a Community Mental Health Center
}

Individuals with serious and persistent mental illness (SPMI) suffer from higher rates of co-morbid medical illness, are twice as likely to develop diabetes, hypertension and obesity and have a life expectancy that is on average 25 years less than the general population. Factors reducing mortality in this population include healthy diet, regular exercise, smoking cessation, and access to primary care. Primary and Behavioral Health Care Integration (PBHCI) is a SAMHSA funded service grant to implement a model of improving access to and engagement with primary care and wellness resources for the adult outpatient population at Worcester's Community Healthlink (CHL). CHL is partnering with the Family Health Center of Worcester in order to provide onsite primary care for this high risk population.

\section{Linking Needs \& Services Through PBHCI Implementation:}

Implementation will include improving access to and participation in care for physical illness, implementing wellness interventions, and improving coordination of care between CHL and primary care providers. Services will include:

- $\quad$ assigning a Nurse Care Manager for program participants

- $\quad$ providing a Nurse Practitioner onsite at CHL with direct access to all medical services of the Family Health Center

- $\quad$ improved systems for tracking blood pressure, weight, blood sugar, lipids, tobacco use, etc

- individual and group counseling regarding tobacco cessation as well as nicotine anonymous meetings

- $\quad$ individual and group walking programs or referrals to local fitness facilities

- $\quad$ nutrition education and mindfulness based stress reduction programs

\section{Determining the Effectiveness of PBHCI:}

Project evaluation will focus on three main areas:

I. Process Outcomes

- the effectiveness of the planning process and implementation of the interventions

II. System Outcomes

- the proportion of mental health consumers screened for obesity, diabetes, co-occurring substance use disorders, etc.

- the proportion of referrals and completed referrals, and participation in wellness activities

\section{Consumer Outcomes}

- participants' Body Mass Index, blood pressure, blood glucose levels, CO levels, etc

- participants' waist circumference

- participants' improved lipid profiles
Anticipated Outcomes for Community Healthlink \& the Family Health Center of Worcester:

During the course of the study, we anticipate improvements in the areas of:

\section{Wellness Interventions}

- individual plans for participants to increase physical activities as well as improve diet

- individualized curriculum on smoking cessation, mindfulness, disease self-management, nutrition, etc.

\section{Redesign of Medical Record}

- prompts on overdue lab work and the technology to visualize changes over time (i.e. weight, cholesterol)

- working with consumers to assist in maintaining their own medical records

\section{System Improvements}

- generate improvements in referral and communications

- forging better ways to share information and scheduling between outside primary care providers and CHL

Principal Investigator: Marie Hobart, MD, Chief Medical Officer, Community Healthlink, Assistant Professor of Psychiatry, UMMS Evaluator: UMass Center for Mental Health Services Research, Funder: Substance Abuse and Mental Health Services Administration Contact: Marie Hobart, MD. Telephone: 508-860-1025, Email: MHobart@communityhealthlink.org 